\title{
A MAGNETORESISTIVE HEAT SWITCH FOR THE CONTINUOUS ADR
}

\author{
E.R. Canavan, M.J. Dipirro, M. Jackson, J. Panek, P.J. Shirron, J.G. Tuttle \\ Cryogenics and Fluids Branch, NASA - Goddard Space Flight Center, \\ Greenbelt, MD 20771
}

In compensated elemental metals at low temperature, a several Tesla field can suppress electronic heat conduction so thoroughly that heat is effectively carried by phonons alone. In approximately one $\mathrm{mm}$ diameter single crystal samples with impurity concentrations low enough that electron conduction is limited by surface scattering, the ratio of zerofield to high-field thermal conductivity can exceed ten thousand. We have used this phenomenon to build a compact, solid-state heat switch with no moving parts and no enclosed fluids. The time scale for switching states is limited by time scale for charging the magnet that supplies the controlling field. Our design and fabrication techniques overcome the difficulties associated with manufacturing and assembling parts from single crystal tungsten. A clear disadvantage of the magnetoresistive switch is the mass and complexity of the magnet system for the controlling field. We have discovered a technique of minimizing this mass and complexity, applicable to the continuous adiabatic demagnetization refrigerator.

\section{INTRODUCTION}

Heat is carried in metals by both electrons and lattice vibrations (phonons). At low temperatures, the electron thermal conductivity is linear with temperature, while phonon thermal conductivity drops off with a higher power, and is typically several orders of magnitude smaller at liquid helium temperatures. A magnetic field suppresses the electron conduction in the plane perpendicular to the field. In metals that are compensated (those having an equal number of holes and electrons) and that have closed Fermi surfaces, the thermal conductivity drops with field until phonon conduction dominates. Metals for which this is true include $\mathrm{Ga}, \mathrm{Cd}, \mathrm{Be}, \mathrm{Zn}, \mathrm{Mo}$, and W. Gorter and Miedema [1] realized that this effect could be used to make a low temperature heat switch, and implemented such a switch [2] with single-crystal gallium. In addition to its inconveniently low melting point, $\mathrm{Ga}$ has a rather high superconducting transition temperature, $T_{c}$. For temperatures below $T_{c}$ thermal conductivity in the on (zero field) state drops as $e^{-T / T_{C}}$, severely restricting the on-off ratio of the switch. If we are interested in a switch that operates well into the 
subkelvin range, the choice of materials is quite limited. Radebaugh [3] pointed out that Be is perhaps the best material for a switch not only because of its extremely low $T_{c}(26 \mathrm{mK})$, but also because it has the highest DeBye temperature, $\Theta_{D}$, of any metal $(1000 \mathrm{~K})$. A high $\Theta_{D}$ implies a very low phonon thermal conductivity, and indeed Radebaugh's measurements indicate that a Be switch would have a useful $\left(>10^{3}\right)$ on-off ratio up to $30 \mathrm{~K}$. Unfortunately, it is very difficult to find large single Be crystals. Of the remaining candidates, tungsten stands out because of its low $T_{c}(15 \mathrm{mK})$ and reasonably high $\Theta_{D}(310 \mathrm{~K})$.

An important application for low temperature heat switches is in adiabatic demagnetization refrigerators (ADRs). ADRs have a number of advantages that make them ideal coolers for space applications. including high efficiency and independence from gravity (which allows full ground testing.) However, in a standard ADR a magnetoresistive heat switch would require a rather large magnet, to put it into the off state at the proper time. Because mass is such an important criterion for a spaceflight instrument, this requirement puts the magnetoresistive switch at a severe disadvantage relative to alternatives.

The standard ADR has a problem of its own: to spend a large fraction of its cycle absorbing heat at the operating temperature, the salt pill must be rather large. Simple scaling of current designs, such as the XRS ADR [4], to meet the requirements of future missions leads to systems with unreasonable size and mass. Recently, Shirron, et al. [5] have circumvented this limitation with the invention of the continuous ADR (CADR). A CADR is a multistage ADR with the stages connected in series. Stage 1, which is attached to the load, acts as a normal ADR during demagnetization; the field is ramped down just fast enough to absorb the heat of the load. However, before this stage reaches zero field, stage 2 is demagnetized to a temperature below the operating temperature and a heat switch between them is closed. At this point, the stage 1 must magnetize to maintain the operating temperature. Once the stage 1 reaches full field, the heat switch is open and it starts demagnetizing again. When the heat switch opens, stage 2 is ramped up. Simultaneously, stage 3 is ramped down. When the temperature of stage 2 rises above that of stage 3 , the heat switch between them is closed, and stage 2 transfers its heat to stage 3 . This cascading of heat from one stage to the next may be repeated by adding additional stages to raise the final heat sink temperature. The key point is that by decoupling the operation of the device being cooled (typically detectors) from the operation of the ADR, the ADR may be designed to cycle much more rapidly, thus providing higher cooling power unit mass.

Note that for any pair of stages in the CADR, the magnetic field of the higher stage is low when the switch needs to be closed, and it is high when the switch needs to be open. Suppose the part of the thermal link between the two pills that is exposed to the magnetic field of the higher stage magnet is a magnetoresistive material. Then it appears that the material will act as a "passive" switch, opening and closing at the proper phase in the cycle without a separate control circuit.

\section{MAGNETORESISTIVITY OF TUNGSTEN}

Tungsten has a simple body centered cubic crystal structure. Because of this, if the field is applied along a high symmetry direction (e.g. 001), the thermal conductivity tensor will have the form

$$
\lambda=\left(\begin{array}{ccc}
\lambda_{x x} & \lambda_{x y} & 0 \\
-\lambda_{y x} & \lambda_{x x} & 0 \\
0 & 0 & \lambda_{z z}
\end{array}\right)
$$


Measurements of the transverse conductivity $\lambda_{x y}$ by Long [6] and Fletcher [7] indicate that it is never more then a few percent of $\lambda_{x x}$ over the range of temperatures and fields of interest. Thus, thermal conductivity is isotropic in the plane normal to the field to within this error.

These and other authors [8], [9] have investigated the electrical and thermal resistivity of single crystal tungsten at helium temperatures and fields up to several Tesla. All find that the component $\lambda_{x x}$ has the form

$$
\lambda_{x x}=\lambda_{g}(T)+\frac{A_{x x}(T)}{B^{2}}
$$

where $\lambda_{\mathrm{g}}$ is the phonon conduction term. Wagner [8] found that in high field, $A_{x x}$ fits to

$$
\frac{A_{\mathrm{xr}}(T)}{T}=\alpha_{0}+\alpha_{3} T^{3} \text {. }
$$

Fitting to his data for a very high quality $(R R R=63,000)$ crystal gives $\alpha_{0}=$ $0.42 \mathrm{Tesla}^{2} \mathrm{~W} \mathrm{~m}^{-1} \mathrm{~K}^{2}$ and $\alpha_{3}=0.0063 \mathrm{Tesla}^{2} \mathrm{~W} \mathrm{~m}^{-1} \mathrm{~K}^{-5}$. The temperature independent part, due to impurity and surface scattering, is related to the temperature independent part of the electrical resistivity, by $\alpha_{0} \approx L_{0} B^{2} / \rho_{x x 0}$, where $L_{0}$ is the Lorenz number.

Long [10] and Wagner [8] find that $\lambda_{\mathrm{g}}$ is proportional to $T^{2}$ with constants of proportionality 0.038 and $0.050 \mathrm{~W} \mathrm{~m}^{-1} \mathrm{~K}^{-1}$, respectively. This result is unusual in two ways: the dependence is quadratic, rather than cubic, as is typically found in dielectrics, and the values in tungsten crystals are far lower than one would expect in a good dielectric crystal. Long shows that this is because phonons are scattering primarily off conduction electrons.

In zero field, the thermal resistivity, $w=k^{-1}$, at low temperature is given by

$$
w T=(w T)_{T=0}+\alpha T^{2}
$$

which has the same form as electrical resistivity, $\rho(\mathrm{T})=\rho_{0}+A T^{2}$. Again, the constant term, $(w T)_{T=0} / \rho_{0} \approx L_{0}$, as one would expect from impurity and surface scattering. On the other hand, Wagner et al. [11] find that $\alpha / A$ is much smaller than $L_{0}$. For the same sample used to derive $\alpha_{0}$ and $\alpha_{3},(w T)_{T=0}=0.00316 \mathrm{~K}^{2} \mathrm{~cm} / \mathrm{W}$ and $\alpha=2.21 \times 10^{-4} \mathrm{~cm} / \mathrm{W}$.

Unfortunately, there is no good theory explaining the low field region. For the purposes of modeling the behavior throughout the range of fields, we assume the magnetothermal resistance acts in parallel with the zero-field resistance:

$$
k(T, B)=k_{g}(T)+\frac{1}{\frac{B^{2}}{A_{x x}(T)}+\frac{(w T)_{T=0}+\alpha T^{2}}{T}} .
$$

Using the above coefficient values in this equation, one finds that zero field to high field thermal conductivity ratios of over $10^{5}$ are possible for $T<2 \mathrm{~K}$ and $B>2 \mathrm{Tesla}$. Unfortunately, for heat switches in the CADR, high thermal conductance is needed at low temperatures, and in the "off" (low conductance) state the switch must span a large temperature range, in which case the conductance is dominated by the thermal conductivity of the high temperature end. The important quantity, the ratio of the heat flows in the two states, is given by the ratio of the integrated thermal conductivity. At high fields (although not so high that $k \approx k_{g}$ ) and low temperatures,

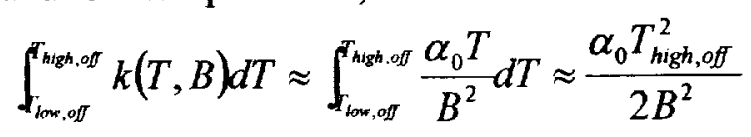

since typically $T_{h i g h, o f f}^{2} \gg T_{l o w, o f f}^{2}$. For zero field at low temperature,

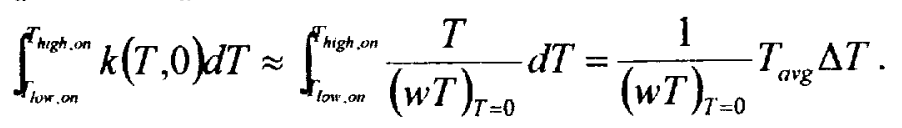


Thus, at low temperatures, the ratio of heat flows is given approximately by

$$
\frac{\dot{Q}_{\text {on }}}{\dot{Q}_{\text {off }}} \approx 2 \frac{B^{2}}{(w T)_{T=0} \alpha_{0}} \frac{T_{\text {avg,on }}}{T_{\text {high }, \text { off }}} \frac{\Delta T_{\text {on }}}{T_{\text {high off }}} \text {. }
$$

This has the advantage that the parameters $(w T)_{T=0}$ and $\alpha_{0}$ can be estimated from electrical resistivity measurements. For the values given above, $\sqrt{(w T)_{T=0} \alpha_{0}}=3.65 \mathrm{mTesla}$. For a typical case: $T_{\text {high,on }}=0.25 \mathrm{~K}, T_{\text {low, on }}=0.225 \mathrm{~K}, T_{\text {high,off }}=1.3 \mathrm{~K}, T_{\text {low,off }}=0.045 \mathrm{~K}, B=$ 1.0 Tesla, this equation gives a ratio of 472 , whereas integration of $\mathrm{Eq}(5)$ gives 529 . Integration must be used at higher temperature; for the case $T_{\text {high,on }}=1.3 \mathrm{~K}, T_{\text {low, on }}=1.2 \mathrm{~K}$, $T_{\text {high,off }}=4.2 \mathrm{~K}, T_{\text {low, off }}=0.225 \mathrm{~K}, B=2.0$ Tesla, it yields a ratio of $1.39 \times 10^{3}$.

For the CADR, heat leak through the switch in the off state, $\dot{Q}_{o f f}$, enters the colder salt pill at $T_{\text {low.off }}$ and must be rejected by heat flow in the on state, $\dot{Q}_{o n}$, at $T_{\text {high,on}}$. Assuming that the pill spends half the cycle period, $\tau$, at $T_{\text {low,off }}$ and half at $T_{\text {high,on, }}$, and that the cycle efficiency is $100 \%$ of Carnot, to do useful cooling, we must have

$$
\dot{Q}_{\text {off }} \frac{\tau}{2} \frac{T_{\text {high,on }}}{T_{\text {low }, \text { off }}}<\dot{Q}_{\text {on }} \frac{\tau}{2}
$$

In fact, the left side, the enthalpy rejected in the on state due to heat leak in the off state, should be a small fraction of the right side, the total enthalpy rejected through the switch in the on state. If we demand that it be no more than $10 \%$, then in the high and low temperature examples above we find requirements of $\dot{Q}_{o n} / \dot{Q}_{o f f}>56$ and 58 , respectively. Thus, very high purity tungsten easily meets this requirement in the two example cases.

\section{SWITCH DESIGN}

The area-to-length ratio of the switch, $A / l$, is set by the requirement that all the heat absorbed in the lower stage when it is at $T_{\text {low, of }}$ be rejected through the switch. Again assuming a $50 \%$ duty cycle and $100 \%$ efficiency, this condition is the same as Eq. (9) with $\dot{Q}_{\text {off }}$ replaced by the total heat load on the lower stage. The on state heat flow is just $A / l \int_{\mathrm{l}_{\mathrm{im}, \text { on }}}^{T_{\text {high,on }}} k(T, 0) d T$. Using a load of $25 \mu \mathrm{W}$ and the temperatures in the previous example, and allowing a factor of two margin to account for inefficiencies gives $A / l=5.2 \times 10^{-6} \mathrm{~m}$.

In experiments on samples of very high purity $(R R R \approx 75000)$, Batdalov [9] found the electron mean free path, $d \approx 1.4 \mathrm{~mm} T=4 \mathrm{~K}$. Reducing the dimensions of the conductor below $d$ significantly increases the effective resistivity. Further, making the width and thickness of the conductor very small also makes the structure overly fragile. For these reasons, a square cross section of $1.5 \mathrm{~mm}$ was chosen. This implies that an effective length of $0.43 \mathrm{~m}$ is required.

As mentioned in the introduction, the key to making the magnetoresistive switch attractive for use in a CADR is that it can exploit the "existing" field to turn the switch off. However, this is not quite true. To accommodate the switch, the magnet must be longer or, equivalently, the salt pill must be smaller. This runs counter to the prime advantage of the CADR: its high cooling power per unit mass. Thus, we need to minimize the length of magnet bore that the switch occupies.

Our solution is shown in FIG 1. Starting with the largest available tungsten single crystal, we wire EDM (electronic discharge machine) a disk $20 \mathrm{~mm}$ in diameter and 5.1 $\mathrm{mm}$ thick. The cylinder $(\mathrm{z})$ axis is aligned with the 001 direction. The other crystal direc- 

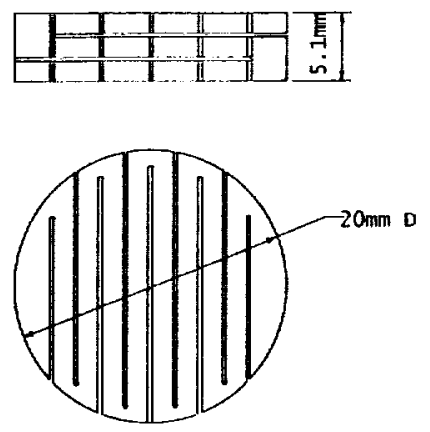

FIGURE 1. Design of magnetoresistive heat switch. The shape is wire EDM cut from a tungsten single crystal.

tions were not known, but, as discussed above, conductivity in the xy plane should be nearly isotropic. The stated purity was $99.995 \%$. Two slots are then cut in the horizontal (xy) plane, and 9 in the $x z$ plane, as shown. The result is effectively a $1.5 \mathrm{~mm} \times 1.5 \mathrm{~mm}$ rod, approximately $0.4 \mathrm{~m}$ long, including only the length of the straight regions. A flexible thermal strap of high purity copper wires is vacuum brazed to the " $D$ " shaped end section.

\section{MEASUREMENTS}

We used x-ray diffraction to characterize the structure of the crystal. A small sample taken from a wire EDM machining test was measured. Nearly all of the power appeared in a single Bragg peak, indicating the material was indeed a single crystal. The approximately one percent of power that appeared randomly aligned was attributed to small surface crystallites caused by rapid remelting during wire EDM machining.

To measure the magnetoresistance of the material, a piece similar to the one shown in FIG.1, but with only a single layer, was cut from the crystal. Four copper leads were vacuum brazed to the sample. At room temperature, the resistance of the sample was 0.0035 Ohm. Using Long's [10] value for room temperature resistivity of pure tungsten, $5.5 \mathrm{mi}$ croOhm-cm, gives $A / l=1.610^{-5} \mathrm{~m}$, which is $7 \%$ larger than that calculated, considering only the straight sections of the sample. The sample was mounted on a probe that fit in the bore of a Supervarimag cryostat. A 0.1 Amp peak-to-peak ac current was applied and the voltage was read on with a lock-in amplifier. Measurements were made at $T=4.26$ $\mathrm{K}$. The resulting resistivity as a function of field is shown in FIG 2 . The zero-field resistance was so low that parasitic reactances dominated the signal into the lock-in. Using a 2 Amp de current and a sensitive voltmeter, we were able to place a lower bound on the zero field resistance of 0.5 microOhm, implying a lower bound of $7 \times 10^{3}$ on the RRR.

Thermal conductance of the material was measured as a function of field using an earlier version of the switch. The geometry was the same as shown in FIG. 1, but the bottom layer was much thicker than the two "active" layers. A ring of commercially pure $(99.95 \%)$ tungsten was brazed around the bottom layer creating an approximately isothermal surface, to which a ruthenium oxide thermometer was varnished. Two bare ruthenium oxide chips were varnished onto the slender arm of the other side of the switch. One was used as a heater. The other was calibrated in situ and used as a thermometer. The switch was placed in a vacuum can that fit into the Supervarimag. A heavy copper braid was used to link the bottom side of the switch to the flange of the vacuum can. 


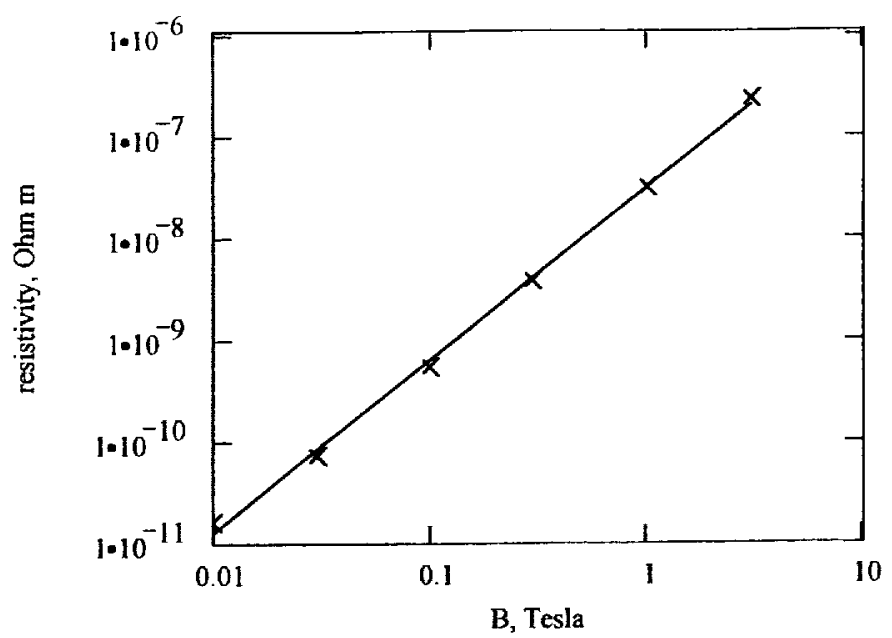

FIGURE 2. Resistivity as a function of field. The solid line is a power law fit.

The thermal resistivity, $w$, times temperature verses magnetic field is plotted in FIG 3 for several different temperatures. The data fit well to a power law: $w T=w T_{1} B^{\beta}$ with $\beta=$ 1.7. The fact that the exponent is not 2 is somewhat surprising. However, the electrical resistivity verses magnetic field data also fits to a power law with an exponent of 1.7 . The two samples have substantially different geometries. Long points out that his data actually fits best to an exponent of 1.96. Fitting the coefficients $w T_{1}$ to the form of EQ.(3) yields $\alpha_{0}$ $=1.37 \mathrm{~W}^{\text {Tesla }}{ }^{1.7} \mathrm{~m}^{-1} \mathrm{~K}^{-2}$ and $\alpha_{3}=0.0061 \mathrm{Tesla}^{2} \mathrm{~W} \mathrm{~m}^{-1} \mathrm{~K}^{-5}$. This value of $\alpha_{3}$ is essentially the same as obtained from Wagner's data. The value of $\alpha_{0}$ is larger, as one might expect from material with higher impurity content. Fitting the zero thermal resistance verses temperature to the form of EQ.(4) yields $(w T)_{T=0}=0.029 \mathrm{~K}^{2} \mathrm{~cm} / \mathrm{W}$ and $\alpha=2.3 \times 10^{-4} \mathrm{~cm} / \mathrm{W}$.

These coefficients can be used to compute $\dot{Q}_{o n} / \dot{Q}_{\text {off }}$ for this material. Substituting the coefficients into EQ.(5), changing the exponent on $B$ to the measured value, and integrating yields ratios of 17 and 78 for the high and low temperature cases discussed previously. In the low temperature case, the ratio does not meet the requirement. A field $>2$ Tesla, higher than desirable for this temperature range, or a higher purity material is needed. However, the ratio in the high temperature case exceeds the requirement (58) by a significant margin.

\section{MODELING SYSTEM PERFORMANCE}

A simple ADR model was modified to simulate a two stage ADR with a "passive" magnetoresistive switch. That is, the field applied to the switch is assumed to be the same as that applied to the higher temperature ("upper") salt pill, which is also connected to a fixed-temperature bath through a gas-gap heat switch. The on conductance of the gas gap switch is realistically modeled, but off conductance is ignored. The stages below the lower stage are simulated with a fixed heat load if the pill temperature was near its lower setpoint, and zero otherwise. The entropy and heat capacity of the salt are modeled using the standard equations for simple, non-interacting magnetic ions. Interactions are taken into account by replacing the applied field, $B$, with $\sqrt{B^{2}+b^{2}}$ [12]. The "interaction field", $b$, for various paramagnetic salts is derived from fitting to zero field entropy data. Despite the simplicity of the entropy function, this model works quite well for single stage ADR's, particularly when using salts with small crystal field splitting and low Neel temperature such as FAA (Ferric Ammonium Alum) and CPA (Chromium Potassium Alum). 


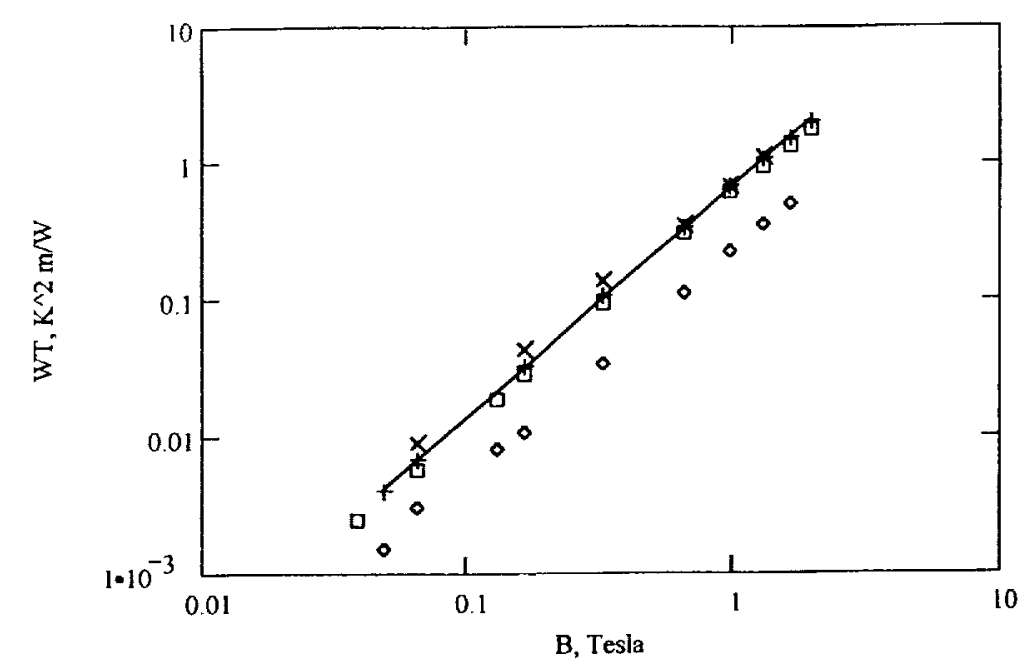

FIGURE 3. Thermal resistivity times temperature of the tungsten sample as a function of magnetic field at several temperatures: $x, 1.6 \mathrm{~K} ;+2.2 \mathrm{~K} ; \square, 4.0 \mathrm{~K} ; \diamond, 8.0 \mathrm{~K}$. The line is a power law fit to the $2.2 \mathrm{~K}$ data.

Simple proportional controllers set the voltage across the magnets. The error signal is to the difference between the temperature and a setpoint. For the lower stage, the algorithm for deciding the setpoint is simple: When the stage is in the magnetizing state, a "high" temperature setpoint is used. Once the magnetic field approaches the maximum allowed value, the stage switches to the demagnetizing state with a "low" temperature setpoint. As the field approaches zero, the stage returns to the magnetizing state. The setpoint algorithm for the upper stage is the same for the magnetizing state. In the demagnetizing state, however, it is somewhat more complex. If the lower stage is still demagnetizing, the setpoint is put at an intermediate value. Although the field on the switch is lower, the temperature difference is also, and a value can be chosen which minimizes heat leak. Once the lower stage is ready to magnetize, the setpoint temperature is calculated to give a fixed heat flow across the switch, unless a minimum temperature is reached.

FIG 4 shows the results of one simulation. In this run, the upper and lower pills were FAA and CPA pills with masses of 150 and $50 \mathrm{gm}$, respectively. The plot shows the third cycle in the simulation, after initial transients have settled out. The first part of the plot is uneventful. The lower stage temperature rapidly reaches its lower setpoint, and the field gradually decays due to the applied heat load. Once the lower stage starts magnetizing, a significant issue for the passive magnetoresistive switch becomes clear: To achieve the desired heat flow in the on state, the upper salt pill must be demagnetized to a temperature far below the upper setpoint of the lower stage. Absorbing the heat at lower temperatures results in lower thermodynamic efficiency. For the cycle shown, the calculated efficiency, as a fraction of Carnot efficiency, is $\left(Q_{\text {load }} / Q_{\text {bath }}\right) \times\left(T_{\text {bath }} / T_{\text {load }}\right)=32 \%$. Approximately one quarter of the efficiency loss is due to heat leak through the magnetoresistive switch in the off state. This can be greatly reduced by operating with a higher maximum field in the upper stage, but at a loss in overall thermodynamic efficiency because the salt pill must drop to a lower temperature.

One way to greatly reduce this loss is to choose a paramagnetic material for the upper stage with an ordering temperature closer to the maximum temperature of the lower stage. In this case, the temperature of the upper stage cannot drop far below that of the lower stage, and the field drops rapidly as the temperature approaches the ordering temperature, allowing the system to take full advantage of the switch's high low field conductance. 


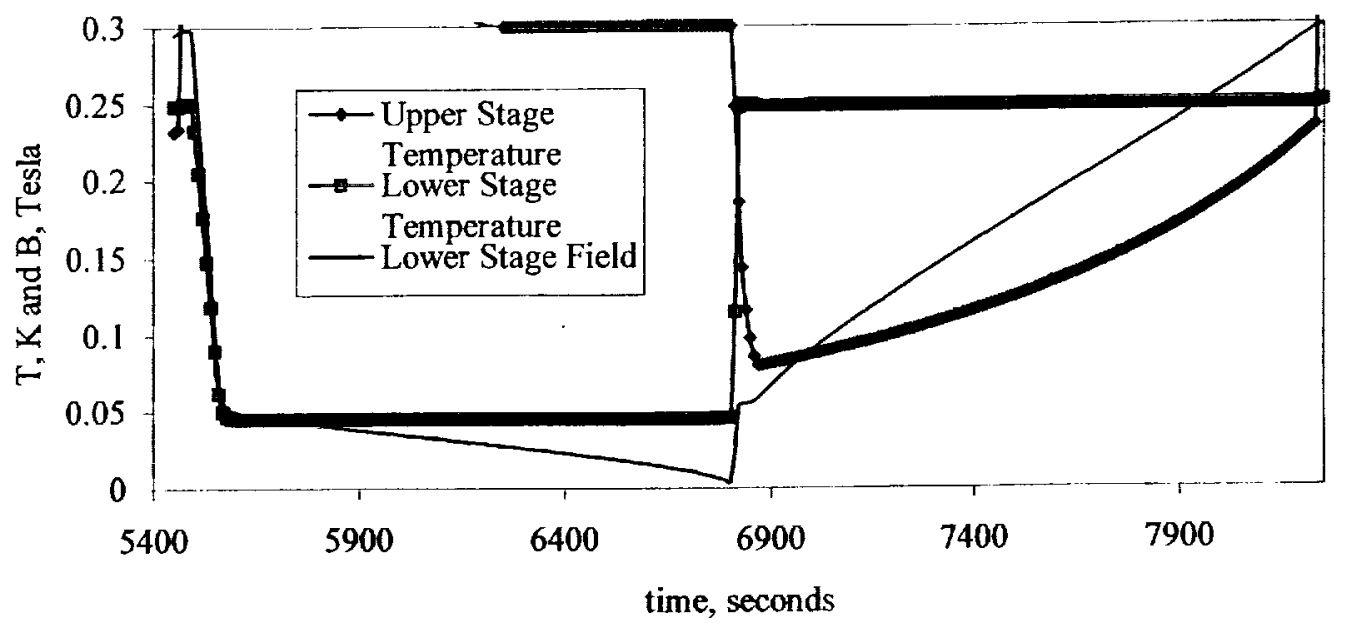

FIGURE 4. Calculated temperature and field behavior for a two stage ADR with a magnetoresitive switch between the stages.

\section{CONCLUSION}

Heat switches based on the thermomagnetoresistance of high purity metals have potential application in ADRs. They are particularly attractive for the continuous ADR because the field of each stage could control the thermal resistance of the switch to the lower stage, thus eliminating the cost and complexity of a separate control magnet. The performance of such a switch, calculated using material properties from the literature, indicate that it should easily satisfy the requirements of the CADR. A switch of the appropriate geometric properties was wire EDM machined from a large tungsten crystals. Measurements on samples cut from this crystal indicate that it will have somewhat lower, although still acceptable, performance. A model was developed to simulate a two-stage ADR using a magnetoresistive switch. Though relatively simple, the model points out that true system performance depends in a complex way on the interactions between the fields, temperatures, and the control algorithm. It shows that if the ordering temperature of the salt in the higher temperature pill is too low, the system will lose efficiency because the pill will need to drop to a lower than desirable temperature to achieve sufficient interstage heat transfer.

\section{REFERENCES}

1 Gorter, F.W., and Miedema, A.R. Cryogenics 8, pp.86 (1968).

2 Engels, J.M.L., Gorter, F.W., and Miedema, A.R. Cryogenics 12, pp.141-145 (1972).

3 Radebaugh, R., Journal of Low Temperature Physics 27, pp. 91-105 (1977).

4 Serlimitsos, A.T., et al., Cryogenics 39(4), pp. 399-404 (1999).

5 Shirron, P.J., et al. Advances in Cryogenic Engineering 45 (2000)

6 Long, J.R., Physical Review B 3(4), pp. 1197-1205 (1971).

7 Fletcher, R., Philosophical Magazine 32, pp. 565-576 (1975).

8 Wagner, D.K., Physical Review B 5(2), pp. 336-347 (1972).

9 Batdalov, A.B., et al. Fizika Tverda Tela 19(3), pp. 672-681 (1977).

10 Long, J.R., Physical Review B 3(8), pp. 2476-2484 (1971).

11 Wagner, et al., Physical Review B 3(10), pp. 3141-3149 (1971).

12 Pobell, F., Matter and Methods at Low Temperatures, Springer-Verlag, Berlin, 1999, Chap. 9. 